\title{
Improvements for drift-diffusion plasma fluid models with explicit time integration
}

\author{
Jannis Teunissen ${ }^{1,2,3}$ \\ ${ }^{1}$ Centrum Wiskunde \& Informatica (CWI), P.O. Box 94079, 1090 GB \\ Amsterdam, The Netherlands \\ ${ }^{2}$ Centre for mathematical Plasma Astrophysics, Department of Mathematics, \\ KU Leuven, Celestijnenlaan 200B, B-3001 Leuven, Belgium \\ ${ }^{3}$ State Key Laboratory of Electrical Insulation and Power Equipment, Xi'an \\ Jiaotong University, Xi'an, China \\ E-mail: jannis@teunissen.net
}

\begin{abstract}
Drift-diffusion plasma fluid models are commonly used to simulate electric discharges. Such models can computationally be very efficient if they are combined with explicit time integration. This paper deals with two issues that often arise with such models. First, a high plasma conductivity can severely limit the time step. A fully explicit method to overcome this limitation is presented. This method is compared to the existing semi-implicit method, and it is shown to have several advantages. A second issue is specific to models with the local field approximation. Near strong density and electric field gradients, electrons can diffuse parallel to the field, and unphysically generate ionization. Existing and new approaches to correct this behavior are compared. Details on the implementation of the models and the various approaches are provided.
\end{abstract}




\section{Introduction}

Simulations of electric discharges are often performed with plasma fluid models [1, 2, 3]. Such models require that the mean free path of electrons is small compared to characteristic length scales of the discharge, so they typically become more accurate at higher pressures (e.g. 1 bar). This paper specifically considers drift-diffusion (DD) models with the local field approximation (LFA), which are referred to as DD-LFA models. Such models are commonly used to simulate e.g., streamer discharges [4, 5, 6, 7, 8, plasma jets [9, 10] and dielectric barrier discharges [11].

Compared to particle simulations, fluid models are often much more computationally efficient [12]. Only a few densities have to be evolved per grid cell, instead of tens or hundreds of particles. Updating these densities in time is relatively cheap when an explicit time integrator is used, with which the state at time $t+\Delta t$ can explicitly be constructed from the previous state(s). The numerical implementation of a DD-LFA model is discussed in section 2 .

However, due to the coupling of charged species to the electric field, an explicit time integrator leads to a restriction on the time step $\Delta t \leq \varepsilon_{0} / \sigma$, where $\varepsilon_{0}$ is the permittivity of vacuum and $\sigma$ the plasma conductivity. This restriction is discussed in more detail in section 3. A new explicit approach is introduced, which avoids the time step restriction by limiting the conductivity of the plasma. The new method is compared to the existing semi-implicit method [13, 14, 15] in several test cases.

Near strong electric field and density gradients, the LFA loses (some of) its validity. An unphysical effect that can occur is that electrons diffuse parallel to the electric field into a high-field region, where they generate ionization. This problem is discussed in detail in section 4. Existing and new approaches to correct this behavior are discussed and tested.

For simplicity, the test cases presented in this paper are one-dimensional. However, the issues addressed are particularly relevant for $2 \mathrm{D}$ and $3 \mathrm{D}$ simulations, which require both high computational efficiency (and thus explicit schemes) and robustness, due to the sharp gradients and the geometrical complexity that can occur.

\section{The DD-LFA model}

In this section, a simple DD-LFA (drift-diffusion with local field approximation) model is introduced. Detailed discussions of the validity of this model, which are outside the scope of the present paper, can be found in e.g. [12, 16, 17, 18, 19, 20, 21. A brief summary is given below.

The accuracy of the LFA depends on the time scale of electron energy relaxation (to the local conditions) compared to other time scales of interest. This energy relaxation occurs through electron-neutral collisions, in particular inelastic ones. In molecular gases at e.g. 1 bar, the LFA therefore works better than in a noble gas at low pressure, if other conditions are kept the same. However, even under favorable conditions, the LFA cannot capture certain (non-local) effects, for example due to spatial density gradients or due to spatial or temporal electric field gradients.

On the other hand, an advantage of the LFA is that only a single equation has to be solved for electrons. Perhaps more important is that the electric field strength is a relatively well-behaved parameter for determining transport coefficients: it is non-negative, well-defined everywhere, and there is a direct link to measured or computed transport coefficients in uniform fields.

\subsection{Model formulation}

The DD-LFA model considered here was chosen to be as simple as possible. Only electrons and a single positive immobile ion species are included

$\partial_{t} n_{e}=-\nabla \cdot \boldsymbol{\Gamma}+S$

$\partial_{t} n_{p}=S$,

where $n_{e}$ is the electron density, $n_{p}$ the positive ion density, $\boldsymbol{\Gamma}$ the electron flux and $S$ the ionization source term. With the drift-diffusion approximation, $\boldsymbol{\Gamma}$ is given by a drift and a diffusion component

$\boldsymbol{\Gamma}=\boldsymbol{\Gamma}^{\text {drift }}+\boldsymbol{\Gamma}^{\text {diff }}=-n_{e} \mu_{e} \boldsymbol{E}-D_{e} \nabla n_{e}$,

where $\mu_{e}$ is the electron mobility, $\boldsymbol{E}$ the electric field vector and $D_{e}$ the electron diffusion coefficient. The electron-impact ionization term is

$S=\bar{\alpha} \mu_{e}|\boldsymbol{E}| n_{e}=\bar{\alpha}\left|\boldsymbol{\Gamma}^{\mathrm{drift}}\right|$,

where $\bar{\alpha}$ is the effective ionization coefficient (i.e., ionization minus attachment), and $|\boldsymbol{E}|$ is the norm of the electric field. With the local field approximation, 


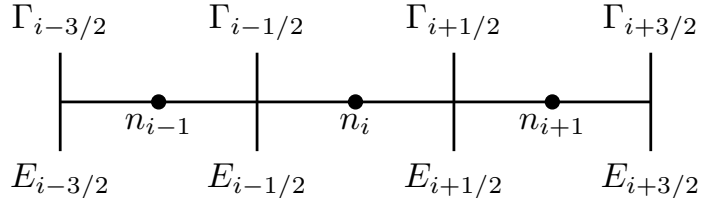

Figure 1. Illustration of a finite-volume discretization in 1D. Densities $n_{i}$ are stored at cell centers. Fluxes $\Gamma_{i+1 / 2}$ and electric fields $E_{i+1 / 2}$ are located at cell faces.

the electron velocity distribution is assumed to be relaxed to the local electric field, so that $\mu_{e}, D_{e}$ and $\bar{\alpha}$ are functions of $|\boldsymbol{E}|$.

For general (multidimensional) simulations, the electric field is computed from the electrostatic potential $\phi$ as $\boldsymbol{E}=-\nabla \phi$, and $\phi$ is obtained by solving a Poisson equation. In simple cases without dielectrics or electrodes, this Poisson equation looks as follows

$\nabla^{2} \phi=-\rho / \varepsilon_{0}$,

where $\varepsilon_{0}$ is the permittivity of vacuum, $\rho=\left(n_{p}-\right.$ $\left.n_{e}\right) e$ the charge density and $e$ the elementary charge. For the 1D simulations considered here, the situation simplifies, as described below.

\subsection{Finite volume implementation}

Fluid simulations of electric discharges are often performed with finite volume (FV) methods, see e.g. [22, 23]. With a FV method, fluxes are computed at the faces of a cell, and the volume-averaged densities inside a cell are updated with these fluxes, see figure 1 .

The flux is here computed using a slope limiter, as described in 24, 25. The idea behind slope limiters is to interpolate cell-centered densities to the cell faces (where the flux has to be computed) in such a way that numerical errors do not grow in time. The scheme's implementation in $1 \mathrm{D}$ is as follows. If $v=-\mu_{e} E_{i+1 / 2}$ is the drift velocity at cell face $i+1 / 2$, then $\Gamma_{i+1 / 2}^{\text {drift }}$ is computed as

$\Gamma_{i+1 / 2}^{\mathrm{drift}}= \begin{cases}v\left[n_{i+1}-\psi\left(1 / r_{i+1}\right)\left(n_{i+1}-n_{i}\right)\right] & v<0 \\ v\left[n_{i}+\psi\left(r_{i}\right)\left(n_{i+1}-n_{i}\right)\right] & v \geq 0\end{cases}$

where $r_{i}=\left(n_{i}-n_{i-1}\right) /\left(n_{i+1}-n_{i}\right)$ and $\psi(x)$ is the limiter function. For brevity of notation, the electron density in cell $i$ is here (and later in the paper) indicated by $n_{i}$. Note that if $\psi(x)=0$, the flux is given by the first-order upwind method. As in [24, 25], the Koren [26] limiter is used, given by

$\psi(x)=\max (0, \min (1,(2+x) / 6, x))$.

The diffusive flux is computed as

$\Gamma_{i+1 / 2}^{\operatorname{diff}}=-D_{e}\left(n_{i+1}-n_{i}\right) / \Delta x$,

where $\Delta x$ is the grid spacing. Note that with the LFA, $\mu_{e}$ and $D_{e}$ depend on $|\boldsymbol{E}|$ at the cell face. In 1D, one can simply take the absolute value, but in multiple dimensions this requires some type of interpolation for the extra components, see e.g. [25].

Unless specified otherwise, source terms are evaluated at the cell centers. Equation (3) is then implemented as

$S_{i}=\bar{\alpha} \mu_{e}\left|E_{i}\right| n_{i}$

where $\bar{\alpha}$ and $\mu_{e}$ depend on the electric field strength at the cell center $\left|E_{i}\right|$, which is here computed as $\left|E_{i}\right|=\left|E_{i-1 / 2}+E_{i+1 / 2}\right| / 2$. As will be shown in sections 3 and 4 it is sometimes beneficial to evaluate the source term at cell faces using the electron flux. On a grid with square cells (e.g., $\Delta x=\Delta y$ ), equation (3) can then be implemented as

$S_{i}=1 / N \sum \bar{\alpha}_{\text {face }}\left|\Gamma_{\text {face }}^{\text {drift }}\right|$,

where the sum runs over all $N$ cell faces, $\bar{\alpha}_{\text {face }}$ depends on the electric field at the cell face, and $\Gamma_{\text {face }}^{\text {drift }}$ is the drift flux through the cell face.

Time integration is here performed with the explicit trapezoidal rule, as in 24, 25]. If equation (1) is written as $\mathbf{y}^{\prime}(t)=\mathbf{f}(\mathbf{y})$, then this scheme is given by

$\tilde{\mathbf{y}}_{t+1}=\mathbf{y}_{t}+\Delta t \mathbf{f}\left(\mathbf{y}_{t}\right)$

$\mathbf{y}_{t+1}=\mathbf{y}_{t}+\frac{\Delta t}{2}\left[\mathbf{f}\left(\mathbf{y}_{t}\right)+\mathbf{f}\left(\tilde{\mathbf{y}}_{t+1}\right)\right]$.

Note that this is an explicit scheme, i.e., that $\mathbf{y}_{t+1}$ can explicitly be computed from $\mathbf{y}_{t}$. Additionally, the classic fourth-order Runge-Kutta time integrator (RK4) is used for some of the convergence tests in section 3. For most of the tests performed here, the time step $\Delta t$ is fixed. In other cases, it is determined as

$\Delta t=0.9 \times \min \left(0.5 \tau_{\mathrm{CFL}}, \frac{1}{1 / \tau_{\mathrm{CFL}}+1 / \tau_{\mathrm{D}}}\right)$,

where $\tau_{\mathrm{CFL}}$ and $\tau_{\mathrm{D}}$ are given by the minimum values of $\Delta x / v$ and $\Delta x^{2} /\left(2 D_{e}\right)$, respectively. Another time step restriction related to the plasma conductivity is sometimes required, see section 3 and specifically equation 13.

A new electric field is computed after each (sub)step of the time integrator. In 1D, this is done by starting from a guess for the electric field at the boundary of the domain. The electric fields at all other cell faces can then be obtained from

$E_{i+1 / 2}=E_{i-1 / 2}+\Delta x \rho_{i} / \varepsilon_{0}$

after which the total voltage difference over the domain is determined. Finally, a constant background field is added to ensure the voltage difference becomes the desired applied voltage. 


\section{The dielectric relaxation time}

In a plasma, the movement of charged species is tightly coupled to the electric field. In general, charges move to screen electric fields that are present within the plasma. A characteristic time scale for this screening is the dielectric relaxation time, also known as the Maxwell time [27, 28]

$\tau=\varepsilon_{0} / \sigma$,

where $\sigma$ is the plasma conductivity. For the discharges considered here, the conductivity is given by

$\sigma=e \sum_{k} \mu_{k} n_{k}$,

where $\mu_{k}$ and $n_{k}$ are the species mobilities and densities. Typically, the contribution of the electron mobility $\mu_{e}$ dominates, so that $\sigma \approx e \mu_{e} n_{e}$. In section 3.1. it is shown that for plasma models with explicit time integration, a time step restriction $\Delta t \leq \varepsilon_{0} / \sigma$ is required.

\subsection{Derivation}

Equation (8) is derived along the lines of [28]. First, recall Maxwell-Ampère's equation

$\nabla \times \boldsymbol{B}=\mu_{0}\left(\boldsymbol{J}+\varepsilon_{0} \partial_{t} \boldsymbol{E}\right)$,

where $\boldsymbol{B}$ is the magnetic field, $\mu_{0}$ the permeability of free space and $\boldsymbol{J}$ the electric current density. Since the divergence of a curl is zero, it follows that

$\nabla \cdot\left(\boldsymbol{J}+\varepsilon_{0} \partial_{t} \boldsymbol{E}\right)=0$.

In the electrostatic approximation with $\boldsymbol{B}=0$ it can be assumed that $\boldsymbol{J}$ and $\boldsymbol{E}$ are (anti-)parallel, since the drift flux is generally (anti-)parallel to $\boldsymbol{E}$ and since the same typically holds for the diffusive fluxes. The divergence can then be transformed into a single spatial derivative, and the generic solution of equation 10 is

$\partial_{t} E=-J / \varepsilon_{0}+C$

where $C$ depends on changes elsewhere in the systen $\ddagger$ Plugging in $J=\sigma E+J^{\text {diff }}$, where $J^{\text {diff }}$ are diffusive terms that do not depend on $E$, gives

$\partial_{t} E=-\frac{\sigma}{\varepsilon_{0}} E-J^{\mathrm{diff}} / \varepsilon_{0}+C$.

When systems with the dynamics of equation 12 are numerically integrated with an explicit method, it is typically required that

$\Delta t \leq \varepsilon_{0} / \sigma$,

otherwise errors grow in time. A physical interpretation of this restriction is that for larger time steps the electric field can reverse, and even be amplified in the reverse direction, which leads to growing errors.

$\ddagger$ In $1 \mathrm{D}, C=0$ as long as the boundary conditions do not change in time.

\subsection{Semi-implicit approach}

The time step restriction of equation 13 can be avoided by solving the plasma fluid equations fully implicitly in time. However, obtaining such implicit solutions is usually computationally expensive, in particular in $2 \mathrm{D}$ and $3 \mathrm{D}$, which is in part due to the non-linear coupling with the electric field. For this reason, semi-implicit discretizations [13, 14, 15] have frequently been used. The idea is to first predict the electric field at $t+\Delta t$ by solving a Poisson equation

$-\nabla^{2} \tilde{\phi}=\nabla \cdot \tilde{\boldsymbol{E}}=\frac{1}{\varepsilon_{0}} \sum_{k} q_{k} \tilde{n}_{k}$

where tildes ( $)$ indicate estimates at $t+\Delta t, \phi$ is the electric potential, $q_{k}$ the charge of species $k$ and $\tilde{n}_{k}$ its density. Since source terms create no net charge, densities can predicted using only the flux $\Gamma$, see equation (2):

$\tilde{n}_{k}=n_{k}^{t}+\Delta t \nabla \cdot \boldsymbol{\Gamma}_{k}\left(n_{k}^{t}, \mu_{k}^{t}, D_{k}^{t}, \tilde{\boldsymbol{E}}\right)$,

where the superscript $t$ indicates quantities known at the present time. Combining equations (15), (14) and (2) leads to a variable-coefficient elliptic PDE, which can be solved to obtain $\tilde{\phi}$ and $\tilde{\boldsymbol{E}}$. Afterwards, the fluid equations can be solved using $\tilde{\boldsymbol{E}}$. Such a semi-implicit approach has the following properties:

- The time step restriction of equation 13 is avoided.

- The scheme is first order accurate in time.

- The resulting variable-coefficient elliptic PDE can be solved quite efficiently, but the cost is typically higher than for constant-coefficient cases such as equation (4).

\subsection{Current-limited approach}

Instead of solving equations implicitly or imposing a restriction on the time step $\Delta t$, another option is to limit the conductivity to allow for a larger time step. Equation (13) then becomes

$\sigma \leq \varepsilon_{0} / \Delta t$.

If both sides are multiplied with $\left|E_{j}\right|$, where $E_{j}$ is the $j^{\text {th }}$ component of the electric field, and it is assumed that $\sigma \approx e \mu_{e} n_{e}$, equation 16 can be rewritten as

$\mu_{e}\left|E_{j}\right| n_{e} \leq \varepsilon_{0}\left|E_{j}\right| /(e \Delta t)$.

The left-hand side of equation 17$\rangle$ is equal to $\left|\Gamma_{j}^{\text {drift }}\right|$, where $\Gamma_{j}^{\text {drift }}$ is the $j^{\text {th }}$ component of the electron drift flux, see equation (2). Instead of limiting only the drift component, it is often preferable to limit the total electron flux $\left|\Gamma_{j}\right|=\left|\Gamma_{j}^{\text {drift }}+\Gamma_{j}^{\text {diff }}\right|$, because diffusive fluxes can also be large near strong density gradients. This is here done according to the following expression

$\left|\Gamma_{j}\right| \leq \varepsilon_{0} E^{*} /(e \Delta t)$, 
where $E^{*}$ should also take diffusion into account. If diffusion is dominant, electron drift and diffusive fluxes will quickly balance each other, and a reasonable choice for $E^{*}$ is the electric field at which this occurs. Solving equation (2) for $\left|\Gamma_{j}\right|=0$ gives $\left|E_{j}\right|=D_{e}\left|\partial_{j} n_{e}\right| /\left(n_{e} \mu_{e}\right)$, where $\partial_{j}$ is the derivative in direction $i$. Conversely, if the drift flux is dominant, $E^{*}$ should be set to $\left|E_{j}\right|$, as was done above. As a general expression for $E^{*}$, the maximum of these two cases is used

$E^{*}=\max \left(\left|E_{j}\right|, \frac{D_{e}\left|\partial_{j} n_{e}\right|}{\mu_{e} n_{e}}\right)$.

A robust way to compute $\left|\partial_{j} n_{e}\right| / n_{e}$ at the face between cells $i$ and $i+1$, where the flux is defined in finite volume schemes, is

$\frac{\left|\partial_{j} n_{e}\right|}{n_{e}} \approx \frac{\left|n_{i+1}-n_{i}\right| / \Delta x}{\max \left(n_{i}, n_{i+1}, \epsilon\right)}$

where $\Delta x$ is the grid spacing and $\epsilon$ is a small number to avoid division by zero. Note that the other quantities in equation 190 can simply be reused from the normal flux computation.

3.3.1. Implementation For convenience, the implementation of the suggested scheme in a discharge model is summarized below:

(i) Determine a time step $\Delta t$ without taking the dielectric relaxation time $\tau=\varepsilon_{0} / \sigma$ into account, for example as in equation (7).

(ii) Compute all the components of the electron flux $\Gamma_{j}$ at the cell faces between the grid cells, for example as described in section 2.2 .

(iii) Compute the values of $E^{*}$ at the cell faces, using equations (19) and (20).

(iv) Limit the components of the electron flux $\Gamma_{j}$ so that equation (18) holds. In other words, if $\left|\Gamma_{j}\right|>$ $\varepsilon_{0} E^{*} /(e \Delta t)$, set $\Gamma_{j}=\operatorname{sgn}\left(\Gamma_{j}\right) \varepsilon_{0} E^{*} /(e \Delta t)$, where $\operatorname{sgn}(x)$ is the sign function.

Sometimes, it can be important to resolve electric screening accurately in time, for example when a highly conductive plasma region is forming. In such cases, the $\Delta t$ in step (ii) can temporarily be reduced.

3.3.2. Discussion The current-limited approach limits the transported charge between two adjacent grid cells within a single time step. Per unit area, the transported charge is $\delta_{\sigma}=e \Delta t\left|\Gamma_{j}\right|$, where $\Gamma_{j}$ is the electron flux in direction $j$. From equation (18), it follows that $\delta_{\sigma} \leq \varepsilon_{0} E^{*}$, which corresponds to a change in local electric field satisfying $\left|\delta_{E}\right| \leq E^{*}$. Two cases can be distinguished for $E^{*}$, see equation (19). When $E^{*}=\left|E_{j}\right|$, it follows that the previous field is at most completely screened. When $E^{*}=D_{e}\left|\partial_{j} n_{e}\right| /\left(\mu_{e} n_{e}\right)$, the change in field is limited by the field that would balance drift and diffusive fluxes.
The current-limited scheme can be applied to drift-diffusion models in general, regardless of whether they use the local field approximation. Advantages of the scheme are that:

- The time step restriction of equation (13) is avoided.

- For time steps fulfilling equation $(13)$ the original behavior of a fluid model is restored.

- It requires only a minor modification of the flux computation.

- The computational cost is low, as the scheme is fully explicit.

\subsection{Comparison of current-limited and semi-implicit approach}

The current-limited approach is now compared with the semi-implicit method in a simplified 1D test case. Equations (12) are solved with the source term set to zero and constant transport coefficients $\mu_{e}=$ $0.03 \mathrm{~m}^{2} /(\mathrm{Vs})$ and $D_{e}=0.1 \mathrm{~m}^{2} / \mathrm{s}$. A computational domain of $10 \mathrm{~mm}$ is used, with a grid spacing $\Delta x=$ $20 \mu \mathrm{m}$. The right boundary is grounded, and a voltage of $10 \mathrm{kV}$ is applied at the left boundary. The initial densities $n_{e}$ and $n_{p}$ are $10^{20} \mathrm{~m}^{-3}$ for $4 \mathrm{~mm} \leq x \leq$ $6 \mathrm{~mm}$, and zero elsewhere. With these parameters, the dielectric relaxation time of equation 13 becomes $\tau \approx 18.4 \mathrm{ps}$.

Figure 2 shows the electric field and the electron density at $t=50 \mathrm{~ns}$ for the current-limited and semiimplicit approach using a time step $\Delta t=80 \mathrm{ps}$. For comparison, a reference solution computed with the fourth-order RK4 time integrator and a small time step $\Delta t=0.1 \mathrm{ps}$ is also shown. This reference solution was computed on the same grid, without special treatment for the dielectric relaxation time. The current-limited approach agrees very well with the reference solution, whereas the semi-implicit method predicts larger peaks in the electric field at the boundaries of the initially ionized area. The left side of the electron density profile also has a visibly different shape with the semi-implicit approach.

Figure 3 shows the root-mean-square error in the electric field (compared to the reference solution) versus the time step. When the time step is reduced, both the current-limited and the semiimplicit approach appear to converge to the reference solution, although with quite different convergence rates. The semi-implicit method shows roughly firstorder convergence. Errors are significantly larger than with the current-limited approach, also for time steps larger than the dielectric relaxation time $\tau$, indicated by the vertical dashed line.

For the current-limited method, the order of convergence depends on the time integrator for 

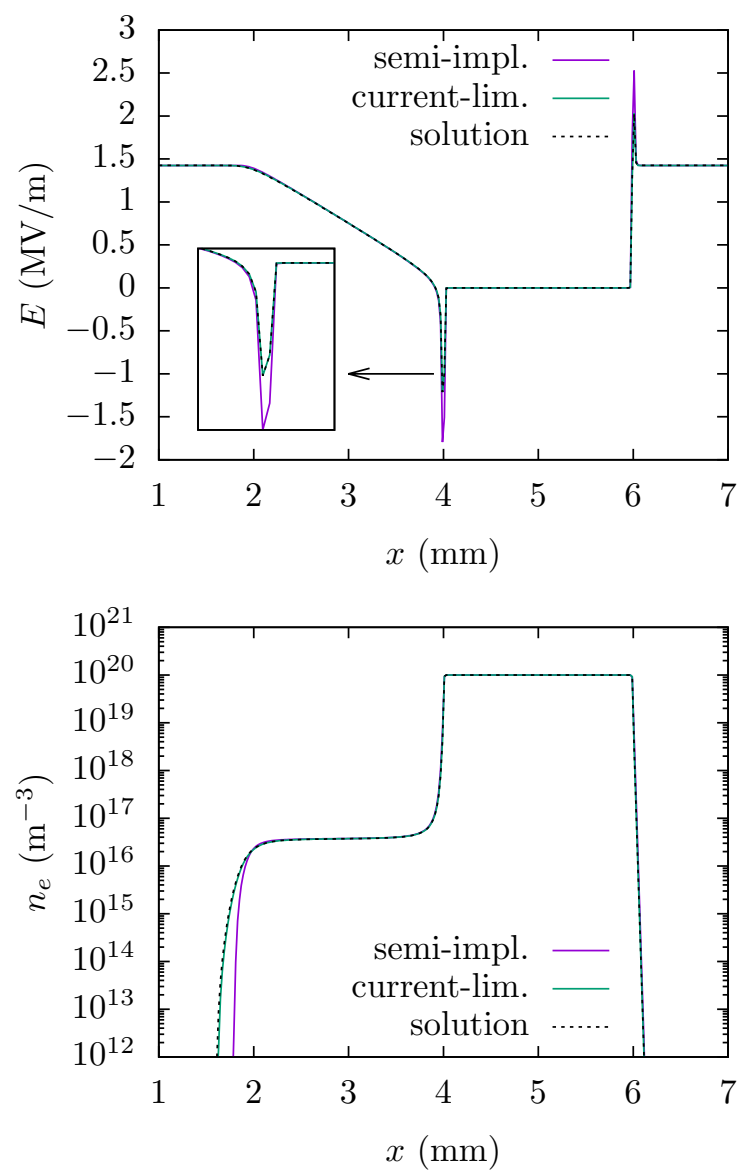

Figure 2. Comparison of the semi-implicit approach with the new current-limited approach. Shown are the electric field (top) and the electron density (bottom) at $t=50 \mathrm{~ns}$ for the test case described in section 3.4 For both approaches, a time step $\Delta t=80 \mathrm{ps}$ is used. A reference solution with a small time step is also shown. With the semi-implicit approach, the electric field has larger peaks at the boundary of the high-density region.

sufficiently small time steps. Two cases are shown: one with the second-order time integrator described in section 2.2, and one with a fourth-order accurate time integrator (RK4). For the latter method, the error saturates for the smallest time steps, which is probably due to numerical round-off errors. For time steps larger than $\tau$, the second and fourth order scheme behave similarly, which indicates that the limiting procedure is the main source of errors.

\subsection{Test in nitrogen}

In this section, the current-limited approach is used in a more realistic test case in nitrogen at 1 bar and $300 \mathrm{~K}$. Transport coefficients and the ionization source term are computed with Bolsig+ [29] as function of the applied electric field, using Phelps' cross sections [30. The same initial condition and grid are used as in section 3.4, but with an applied voltage of $40 \mathrm{kV}$. After

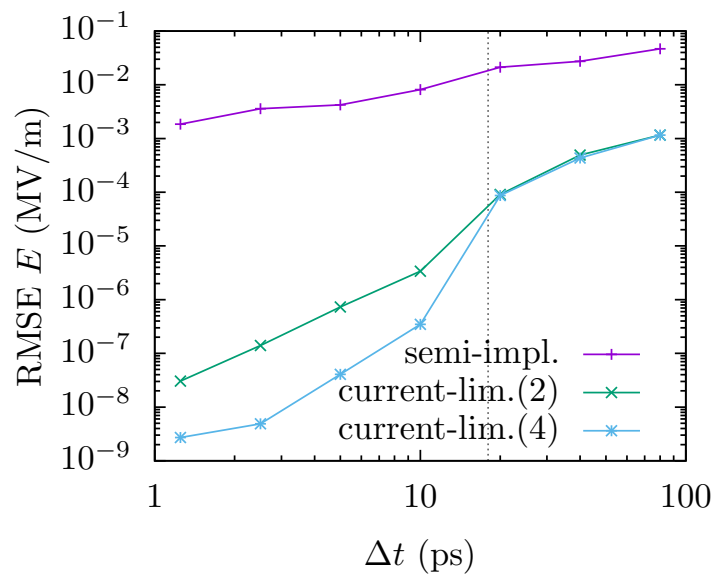

Figure 3. A comparison of the convergence behavior of the semi-implicit and current-limited approaches for the test case described in section 3.4 Shown is the root-mean-square error (compared to the reference solution) in the electric field at $t=50 \mathrm{~ns}$ versus the time step. For the current-limited approach, results are shown with a second-order and a fourth-order accurate time integrator, indicated by (2) and (4), respectively. The vertical dashed line indicates the dielectric relaxation time.

electric screening has taken place in the initial plasma region, the dielectric relaxation time is about $\tau \approx 3 \mathrm{ps}$. This value depends on the highest tabulated electron mobility (for the lowest electric field), which was here $\mu_{e} \approx 0.186 \mathrm{~m}^{2} /(\mathrm{Vs})$.

Figure 4 shows the electron density and electric field in the system at several times. The solid lines show results with the current-limited approach and a fixed time step $\Delta t=20 \mathrm{ps}$. The dashed lines show a reference solution, computed on the same numerical grid but with a small time step $\Delta t=0.1 \mathrm{ps}$ and a fourth-order time integrator (RK4). Visually, the current-limited results are almost identical to the reference solution, indicating that the approach also works well in more realistic test cases. The convergence to the reference solution is shown in figure 5. Second order convergence is obtained, which indicates that the current-limited approach does not affect the convergence of the time integration scheme.

\subsection{Relevance for discharge simulations}

Avoiding the time step restriction due to the dielectric relaxation time $\tau$ is beneficial when $\tau$ becomes smaller than other time step constraints, in particular when $\tau<\tau_{\mathrm{CFL}}$, see equation (7). This can for example happen when:

- High-density low-pressure discharges are simulated, which have a high conductivity, see e.g. [13, 14 .

- A localized high-density and thus high-conductivity region is present, for example near an electrode or 

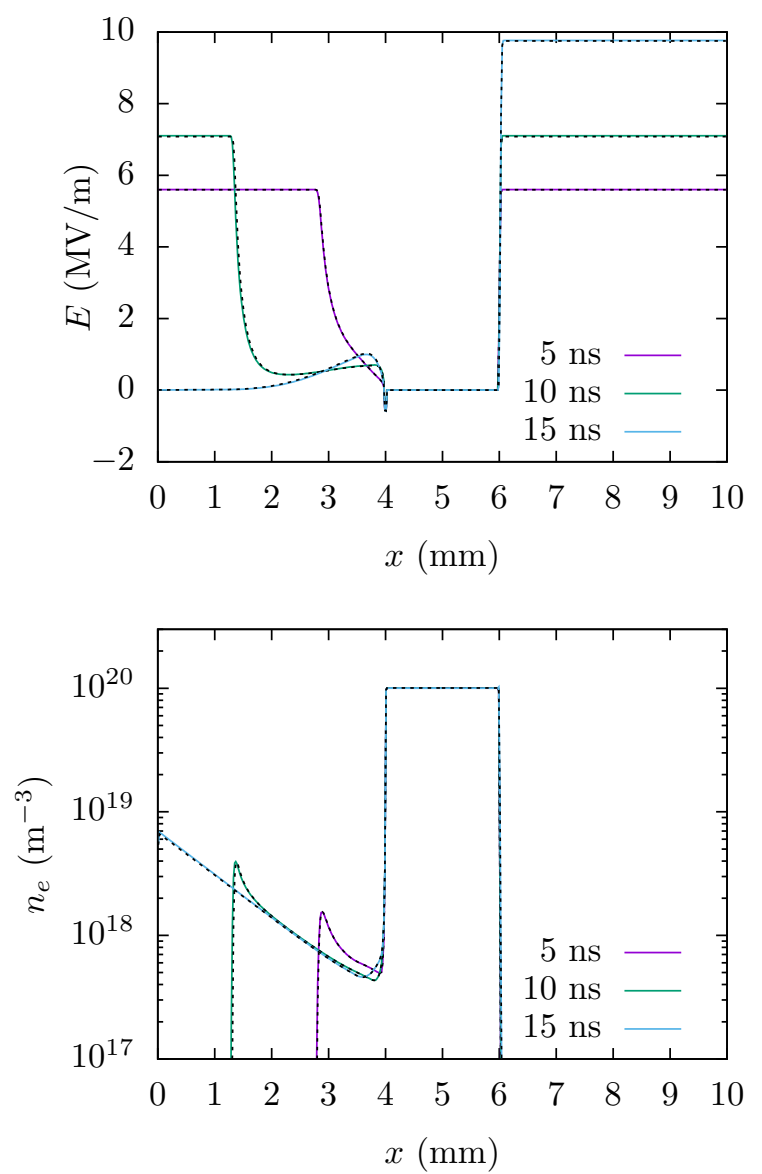

Figure 4. Results of the current-limited approach (solid lines) for a test case in nitrogen, see section 3.5 Shown are the electric field (top) and the electron density (bottom) at several times. The dashed lines indicate a reference solution with a small time step $\Delta t=20 \mathrm{ps}$.

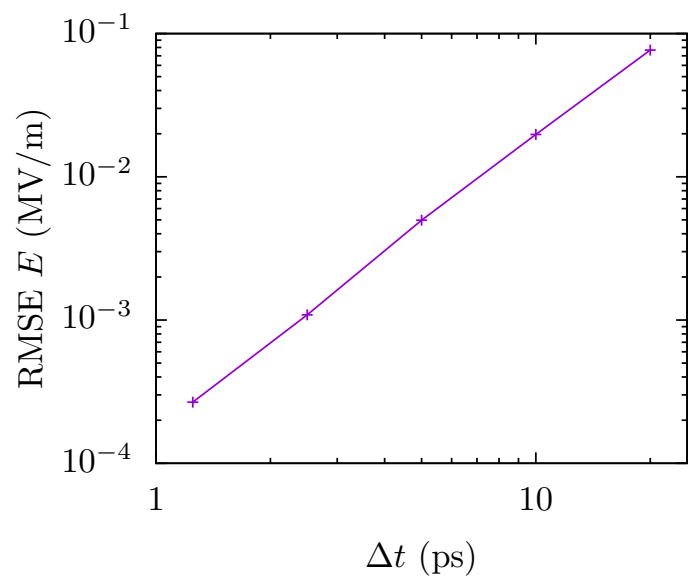

Figure 5. The convergence behavior of the current-limited approach for a test case in nitrogen, see section 3.5 Shown is the root-mean-square error (compared to the reference solution) in the electric field at $t=10 \mathrm{~ns}$ versus the time step. The line indicates that second order convergence is obtained. a dielectric material, see e.g. 31, 32, or when streamer discharges stagnate [33].

- The evolution of a conductive plasma is studied after the voltage has been turned off.

\section{Unphysical diffusion}

The diffusive term in the electron flux of equation (2) can sometimes lead to unphysical behavior, in particular when the diffusive flux has a component parallel to $\boldsymbol{E}$. This can for example happen when there is an electrically screened region with a high electron density, bordered by a region without electrons where the electric field is above breakdown. In reality, electrons should quickly lose energy as they diffuse parallel to the electric field, so that they are effectively confined. However, a fluid model with the LFA does not capture these dynamics, and allows electrons to diffuse into the high-field region $\S$. These electrons then start generating electron-impact ionization, since their ionization rate only depends on the local electric field.

This unphysical effect can for example occur when simulating streamer discharges close to dielectric surfaces $[32,34,35$. The author has also encountered it in 3D simulations of branching streamer discharges [25], in which some of the smaller branches stop to grow 33 .

Below, the unphysical behavior is first demonstrated in a test case. Several approaches to prevent the unphysical behavior are then discussed, and these approaches are compared in section 4.5 .

\subsection{Test case}

The unphysical behavior due to parallel diffusion is present in the test case of section 3.5 but it is not yet visible at $t=15 \mathrm{~ns}$ in figure 4. To demonstrate the unphysical effect more clearly, simulations are here performed in a higher background field. A computational domain of $4 \mathrm{~mm}$ is used with a voltage difference of $40 \mathrm{kV}$, so that the background field of $10 \mathrm{MV} / \mathrm{m}$ points in the $+x$-direction. A grid spacing of $\Delta x=4 \mu \mathrm{m}$ is used. Initially, $n_{e}=n_{p}=10^{20} \mathrm{~m}^{-3}$ in the left half of the domain, and $n_{e}=n_{p}=0$ in the right half of the domain. As in section 3.5, the simulations are performed in nitrogen at 1 bar and $300 \mathrm{~K}$.

The evolution of the electron density is shown in figure 6. which also includes results with half the grid spacing $(\Delta x=2 \mu \mathrm{m})$. In both cases, electrons diffuse to the right of the original plasma boundary. When they enter the high-field region, they generate ionization, extending the plasma to the right. Using a finer grid spacing slows down the unphysical growth of the plasma, but does not prevent it. Not shown here

$\S$ Moreover, a strong parallel electric field will typically give a higher diffusion coefficient with the LFA. 

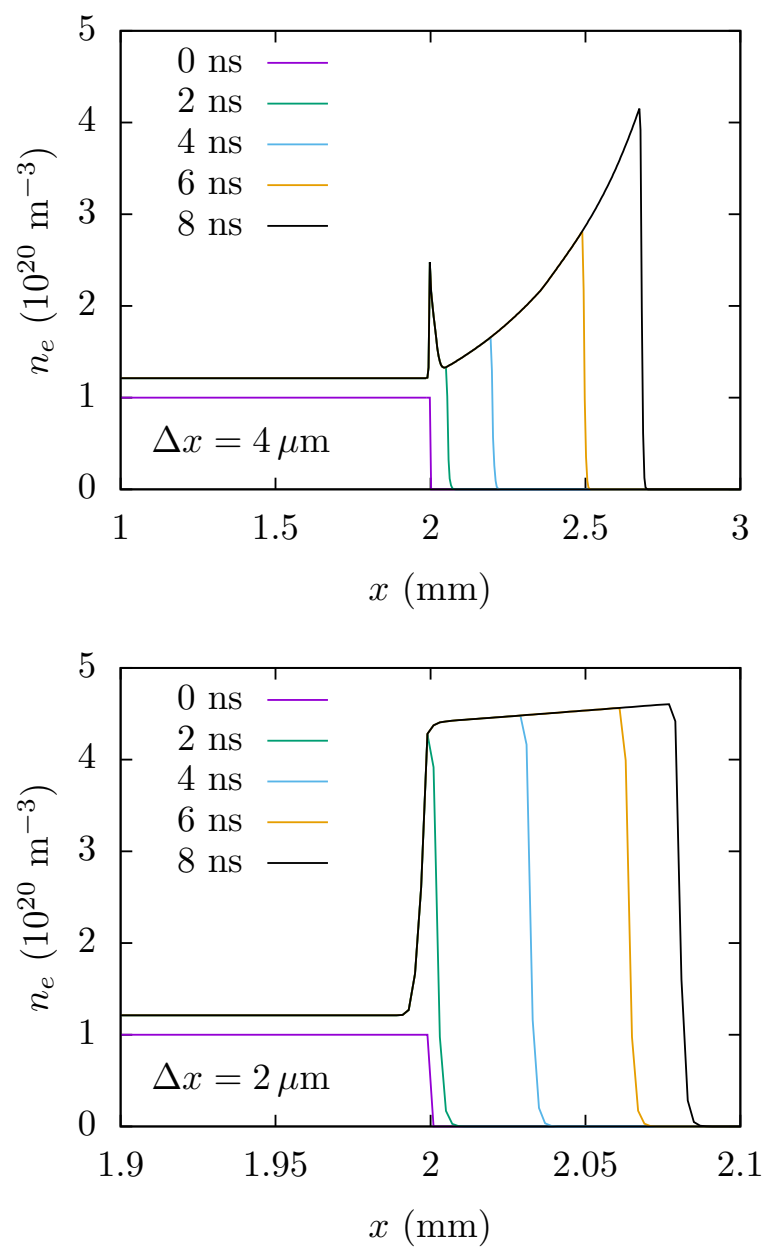

Figure 6. Illustration of the unphysical growth of an ionized region parallel to the electric field, which here points to the right. In the standard DD-LFA model, electrons can diffuse into the high-field region on the right, where they generate impact ionization. The grid spacing for the top and bottom figure are $4 \mu \mathrm{m}$ and $2 \mu \mathrm{m}$, respectively.

is the electric field, which is quickly screened in the left half of the domain [28, after which it doubles in the right half of the domain. During the initial electric screening, the degree of ionization in the left half of the domain slightly increases.

\subsection{Source term factor $\left(f_{\epsilon}\right.$ scheme $)$}

The LFA typically becomes less accurate when there are strong density and/or field gradients [36, 37. Soloviev and Krivtsov 34] derived a correction factor $f_{\epsilon}$ for the impact ionization term from the electron energy equation, which is here referred to as the $f_{\epsilon}$ scheme. This factor can be written as

$f_{\epsilon}=1-\frac{\hat{E} \cdot \Gamma^{\text {diff }}}{\left|\Gamma^{\text {drift }}\right|}=1+\frac{\hat{E} \cdot\left(D_{e} \nabla n_{e}\right)}{\mu_{e} n_{e}|E|}$,

where $\hat{E}$ is the electric field unit vector. Near a strong density gradient such as shown in figure 6. $f_{\epsilon}$ will go to zero, so that no unphysical ionization will take place. This happens because the advective and the diffusive flux balance each other, with the diffusive flux occurring parallel to $\boldsymbol{E}$. Note that equation (21) also gives reasonable results when the drift and diffusive fluxes do not completely cancel. For example, if the diffusive flux balances half the drift flux $\left(\boldsymbol{\Gamma}^{\text {diff }}=\right.$ $-\Gamma^{\text {drift }} / 2$ ), the result is $f_{\epsilon}=1 / 2$.

A downside of using equation (21) is that the original model formulation is modified, also in regions where there are no unphysical effects due to diffusion. Another issue is how to compute $f_{\epsilon}$ numerically. If the right-most expression of equation (21) is used, there are two problems. First, quantities such as $n_{e}, \boldsymbol{E}$ and $\nabla n_{e}$ are not all defined at the same location in a numerical grid cell, so that some type of interpolation or averaging is required. Second, since both $n_{e}$ and $E$ can be small (or even zero), the division can be problematic. However, $f_{\epsilon}$ can robustly be computed at a cell face if the electron flux is used. In 1D, equation (21) can then be implemented as

$f_{\epsilon}=1-\frac{\operatorname{sgn}(E) \Gamma^{\text {diff }}}{\max \left(\left|\Gamma^{\text {drift }}\right|, \epsilon\right)}$,

where all quantities are defined at the cell face, $\operatorname{sgn}(x)$ is the sign function, and $\epsilon$ is a small number to avoid division by zero. In $2 \mathrm{D}$ or $3 \mathrm{D}$, the term $\operatorname{sgn}(E) \Gamma^{\text {diff }}$ would be replaced by $\hat{E}_{j} \Gamma_{j}^{\text {diff }}$, where $\hat{E}_{j}$ is the $j^{\text {th }}$ component of the electric field unit vector, and $\left|\Gamma^{\text {drift }}\right|$ would be replaced by $\left|\Gamma_{j}^{\text {drift }}\right|$.

When $f_{\epsilon}$ is computed at cell faces, it makes sense to also evaluate the source term at cell faces, so that equation (6) becomes

$S_{i}=\left(\bar{\alpha}_{L} f_{\epsilon, L}\left|\Gamma_{L}^{\text {drift }}\right|+\bar{\alpha}_{R} f_{\epsilon, R}\left|\Gamma_{R}^{\text {drift }}\right|\right) / 2$,

where the subscripts $L$ and $R$ indicate values on the left and right face of the cell. In this paper, the $f_{\epsilon}$ scheme is implemented according to equation (23). Alternatively, it is also possible to approximate $f_{\epsilon}$ at the cell center, for example as

$f_{\epsilon}=1-\frac{\operatorname{sgn}\left(E_{L}\right) \Gamma_{L}^{\text {diff }}+\operatorname{sgn}\left(E_{R}\right) \Gamma_{R}^{\text {diff }}}{\left|\Gamma_{L}^{\text {drift }}\right|+\left|\Gamma_{R}^{\text {drift }}\right|+\epsilon}$,

after which the standard source term of equation (5) can be used.

Note that $f_{\epsilon}$ can in principle become negative, which would be unphysical. Here $f_{\epsilon}$ is set to zero in such cases. Similarly, $f_{\epsilon}$ can be restricted to be at most one, to prevent an increase in the source term.

\subsection{Source term from flux (FFS scheme)}

The source term in equation (3) can also be approximated by the full electron flux

$S=\bar{\alpha} \mu_{e}|E| n_{e}=\bar{\alpha}\left|\Gamma^{\mathrm{drift}}\right| \approx \bar{\alpha}|\Gamma|$, 
where $\Gamma$ is given by equation (2). This approach is here referred to as the FFS (Full Flux Source) scheme. The FFS scheme has a similar effect as the factor introduced in equation 21). When the advective and diffusive fluxes balance each other, the source term is zero. The effective factor introduced in equation (24) is $g=|\Gamma| /\left|\Gamma^{\text {drift }}\right|$. When the advective and diffusive flux are (anti-)parallel, which is always the case in $1 \mathrm{D}$, it follows that $g=f_{\epsilon}$. For example, when the fluxes are in opposite directions $g=1-\left|\Gamma^{\text {diff }}\right| /\left|\Gamma^{\text {drift }}\right|$, just as for equation (21). In general $2 \mathrm{D}$ or $3 \mathrm{D}$ cases, $g \neq f_{\epsilon}$. When the advective and diffusive flux are orthogonal, $g=\sqrt{1+\left(\left|\Gamma^{\text {diff }}\right| /\left|\Gamma^{\text {drift }}\right|\right)^{2}}$, whereas $f_{\epsilon}=$ 1. However, in such cases the diffusive flux is typically small compared to the advective flux, so that $g \approx$ $1+\left(\left|\Gamma^{\text {diff }}\right| /\left|\Gamma^{\text {drift }}\right|\right)^{2} / 2 \approx 1$.

Compared to equation 21, there is less physical motivation for equation (24). Both approaches have the drawback that the original model formulation is changed. However, numerically equation (24) is easier to implement than equation 21, since the electron flux is readily available. The source term can be computed similar to equation (6), so that in $1 \mathrm{D}$ it is given by

$S=\left(\bar{\alpha}_{L}\left|\Gamma_{L}\right|+\bar{\alpha}_{R}\left|\Gamma_{R}\right|\right) / 2$,

where $\Gamma_{L}$ and $\Gamma_{R}$ are the flux through the left and right face of the cell. Finally, note that equation (24) can be modified so that it does not increase the source term by replacing $|\Gamma|$ with $\min \left(\left|\Gamma^{\text {drift }}\right|,|\Gamma|\right)$.

\subsection{Limiting parallel diffusion}

With the above two methods, the source term is modified next to a strong density gradient. This prevents electrons that have diffused across the gradient from generating new ionization. Another approach could be to limit diffusion parallel to the electric field, which can be implemented as:

if $\Gamma_{j}^{\text {diff }} E_{j}>0$ and $\left|E_{j}\right|>E_{\text {lim }}$ then set $\Gamma_{j}^{\text {diff }}=0$.

Or in words: if the $j^{\text {th }}$ component of the diffusive flux points in the same direction as $E_{j}$, and if $\left|E_{j}\right|$ exceeds some threshold $E_{\text {lim }}$, set the diffusive flux to zero. By setting $E_{\text {lim }}$ to the critical field, above which the net ionization rate is positive, electrons cannot diffuse into a region where they generate ionization.

Advantages of this approach are that only the diffusion equation has to be modified and that the implementation is relatively simple. However, there are also some downsides. As long as the electron density is not strictly zero, ionization continues to occur when the field is above breakdown. For the implementation described above, it is also necessary to evaluate the source term using the electron drift flux, as in equation (6). Otherwise, electrons can diffuse in from one of the

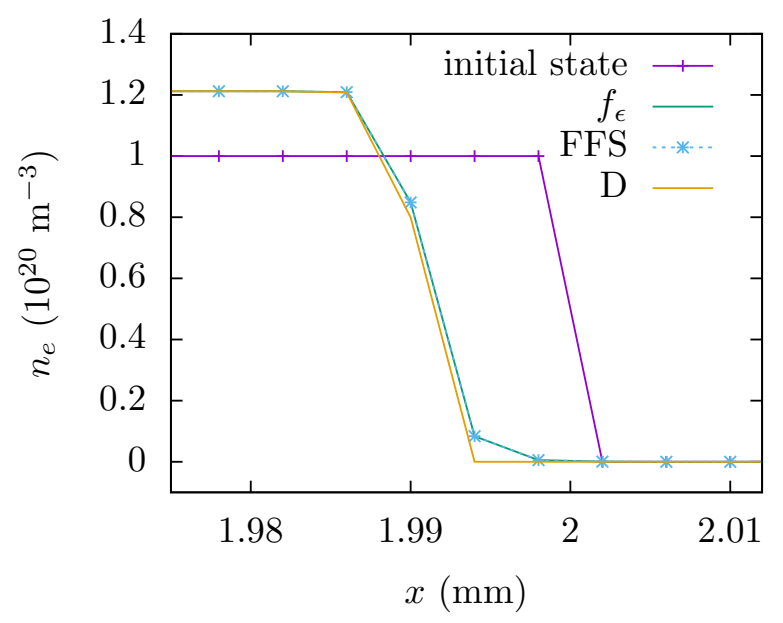

Figure 7. Comparison of methods to address unphysical diffusion, showing the electron density at $t=8 \mathrm{~ns}$ for the test case described in section 4.1 The $f_{\epsilon}$ curve corresponds to equation 21, the FFS curve to equation 24 and the $D$ curve to the diffusion-limited approach described in section 4.4 The $f_{\epsilon}$ and FFS scheme give identical results. All methods prevent unphysical growth of the discharge to the right.

cell faces (with a low field), and generate ionization at the cell center due to a high field on the other cell face.

\subsection{Comparison of the schemes}

In figure 7, the effect of the three approaches described above is compared. Applied to the half-ionized domain test case described in section 4.1, the results at $t=$ $8 \mathrm{~ns}$ show no unphysical propagation to the right. As expected, equation (21) and equation (24) give identical results in 1D. With the diffusion-limited approach, the electron density drops to zero more rapidly. From this $1 \mathrm{D}$ test, it cannot be concluded which scheme will perform best for general 2D and 3D cases; this question is left for future work.

In this paper, several approaches have been described for computing the ionization source term. Their effect on a developing discharge is illustrated in figure 8, which shows results for four cases:

- A standard DD-LFA model, using equation (5) as the source term

- A DD-LFA model that uses the drift flux for the source term, see equation (6)

- The $\epsilon$ and FFS schemes (which give identical results)

- The $\epsilon$ and FFS schemes, modified such that they do not increase the ionization rate

The same computational domain and gas as in section 4.1 are used, but with $30 \mathrm{kV}$ applied voltage. Initially, $n_{e}=n_{p}=10^{10} \mathrm{~m}^{-3}$ for $2.95 \mathrm{~mm} \leq x \leq 3.05 \mathrm{~mm}$, and 


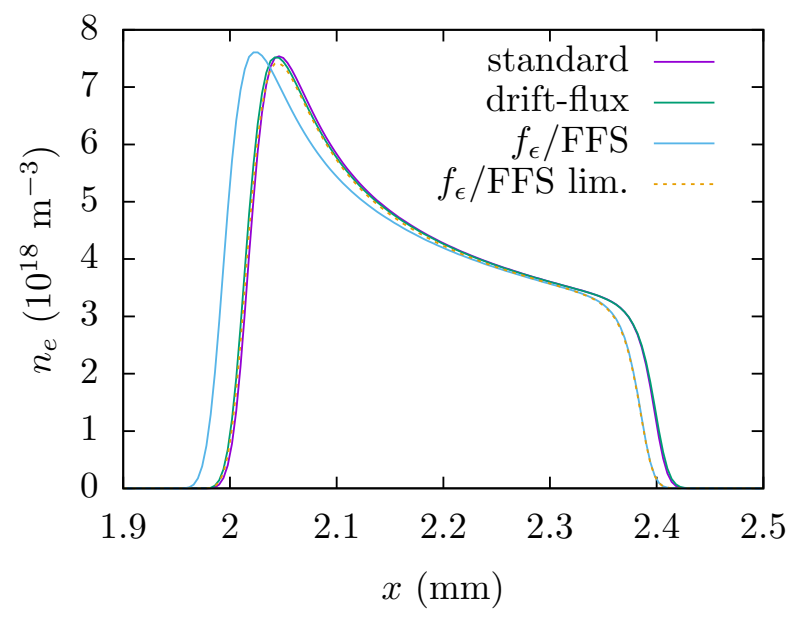

Figure 8. Comparison of several methods to compute the ionization source term for the test case described in section 4.5 The electron density at $t=3 \mathrm{~ns}$ is shown. Using equation (5) for the source term (labeled standard) produces almost the same results as equation (6) (labeled drift-flux). The $f_{\epsilon} /$ FFS schemes can increase the source term, which causes the discharge to propagate slightly faster. When the source is limited (so that it does not exceed its 'normal' value) this effect disappears, as indicated by the dashed line.

zero elsewhere. All simulations were performed with a fixed time step $\Delta t=3 \mathrm{ps}$.

With the $\epsilon /$ FFS schemes, the source term on the right side of the discharge is reduced, leading to a smaller extension in this direction. Conversely, these schemes increase the source term on the left side, leading to faster leftward propagation than with the standard model. Having a higher ionization rate in front of the discharge physically makes sense [20, but the changes introduced by equations (21) and (24) are not necessarily the best way to correct for this. When the $\epsilon /$ FFS schemes are modified so that they do not increase the source term, the results are in good agreement with the standard model for the leftward propagation. Finally, figure 8 shows that equation (5) and equation (6) give almost the same results for this test case.

\section{Summary and discussion}

The main contributions of the present paper are:

- A new explicit approach to avoid the dielectric relaxation time step restriction was presented in section 3. Compared with the existing semi-implicit method, the new current-limited approach is faster, simpler to implement, does not reduce the order of accuracy of the time integrator, and it reverts to the original model for sufficiently small time steps. The proposed current-limited approach can be used with different types of plasma fluid models.

- With the local field approximation, unphysical effects can occur when there is diffusion parallel to the electric field. Several methods to avoid this were compared in section 4. An existing approach [34 was compared with two new approaches. All methods could prevent the unphysical effects in 1D.

- Different methods for implementing the ionization source term were described, as well as implementation details for the approaches listed above. General implementation aspects for explicit driftdiffusion fluid models were also discussed.

The methods described here are particularly relevant for the simulation of pulsed discharges near dielectrics and electrodes. Near such boundaries, there can be high electron densities, strong electric fields, and strong spatial gradients in both quantities, see e.g. 32. For simplicity, tests were here performed in $1 \mathrm{D}$, although the described methods can also be used in $2 \mathrm{D}$ and $3 \mathrm{D}$. Performing these $2 \mathrm{D}$ and/or $3 \mathrm{D}$ tests is an important next step that is left for future work. For the unphysical effects due to diffusion, it would also be interesting to investigate the effect of using an energy equation instead of the local field approximation.

\section{Acknowledgments}

JT was supported by fellowship $12 \mathrm{Q} 6117 \mathrm{~N}$ from Research Foundation - Flanders (FWO) and by the State Key Laboratory of Electrical Insulation and Power Equipment (EIPE18203). Robert Marskar is acknowledged for pointing out reference [34.

\section{Software availability}

The source code of the simulation software used in this paper is available at: https://gitlab.com/ MD-CWI-NL/streamer_1d and https://github.com/ jannisteunissen/semi_implicit_fluid

\section{References}

[1] Gogolides E and Sawin H H 1992 J. Appl. Phys. 7218

[2] Dujko S, Markosyan A H, White R D and Ebert U $2013 \mathrm{~J}$. Phys. D: Appl. Phys. 46475202 ISSN 1361-6463

[3] Alves L L, Bogaerts A, Guerra V and Turner M M 2018 Plasma Sources Science and Technology 27023002 ISSN 1361-6595

[4] Luque A and Ebert U 2012 Journal of Computational Physics 231 904-918 ISSN 00219991

[5] Bagheri B, Teunissen J, Ebert U, Becker M M, Chen S, Ducasse O, Eichwald O, Loffhagen D, Luque A, Mihailova D and al e 2018 Plasma Sources Science and Technology 27095002 ISSN 1361-6595

[6] Marskar R 2019 Journal of Computational Physics $\mathbf{3 8 8}$ 624-654 ISSN 00219991 
[7] Plewa J M, Eichwald O, Ducasse O, Dessante P, Jacobs C, Renon N and Yousfi M 2018 Journal of Physics D: Applied Physics 51095206 ISSN 0022-3727, 1361-6463

[8] Komuro A, Matsuyuki S and Ando A 2018 Plasma Sources Science and Technology 27105001 ISSN 1361-6595

[9] Boeuf J P, Yang L L and Pitchford L C 2013 Journal of Physics D: Applied Physics 46015201 ISSN 0022-3727, 1361-6463

[10] Bourdon A, Darny T, Pouvesle J M, Viegas P and Is S 2016 Plasma Sources Sci. Technol. 18

[11] Stollenwerk L and Amiranashvili S 2006 PHYSICAL REVIEW LETTERS 4

[12] Kim H C, Iza F, Yang S S, Radmilovi \'c-Radjenovi `c M and Lee J K 2005 Journal of Physics D: Applied Physics 38 R283-R301 ISSN 1361-6463

[13] Ventzek P L, Hoekstra R J and Kushner M J 1994 Journal of Vacuum Science \& Technology B: Microelectronics and Nanometer Structures Processing, Measurement, and Phenomena 12 461-477

[14] Lapenta G, Iinoya $\mathrm{F}$ and Brackbill J Aug/1995 IEEE Transactions on Plasma Science 23 769-779 ISSN 00933813

[15] Hagelaar G and Kroesen G 2000 Journal of Computational Physics 159 1-12 ISSN 00219991

[16] Grubert G K, Becker M M and Loffhagen D 2009 Physical Review E 80 ISSN 1550-2376

[17] Becker M M and Loffhagen D 2013 AIP Advances 3012108 ISSN 2158-3226

[18] Markosyan A H, Teunissen J, Dujko S and Ebert U 2015 Plasma Sources Science and Technology 24065002 ISSN 1361-6595

[19] Eichwald O, Ducasse O, Merbahi N, Yousfi M and Dubois D 2005 Journal of Physics D: Applied Physics 39 99-107 ISSN 1361-6463

[20] Li C, Brok W J M, Ebert U and van der Mullen J J A M 2007 Journal of Applied Physics 101123305 ISSN 00218979

[21] Drallos P J, Nagorny V P and Williamson W 1995 Plasma Sources Science and Technology 4 576-590 ISSN 09630252, 1361-6595

[22] LeVeque R J 2002 Finite Volume Methods for Hyperbolic Problems Cambridge Texts in Applied Mathematics (Cambridge ; New York: Cambridge University Press) ISBN 978-0-521-81087-6 978-0-521-00924-9

[23] Toro E F 2009 Riemann Solvers and Numerical Methods for Fluid Dynamics: A Practical Introduction 3rd ed (Dordrecht ; New York: Springer) ISBN 978-3-54025202-3 978-3-540-49834-6 oCLC: ocn401321914

[24] Montijn C, Hundsdorfer W and Ebert U 2006 Journal of Computational Physics 219 801-835 ISSN 00219991

[25] Teunissen J and Ebert U 2017 Journal of Physics D: Applied Physics 50474001 ISSN 1361-6463

[26] Koren B 1993 A robust upwind discretization method for advection, diffusion and source terms Numerical Methods for Advection-Diffusion Problems ed Vreugdenhil C B and Koren B (Braunschweig/Wiesbaden: Vieweg) pp $117-138$

[27] Barnes M S, Cotler T J and Elta M E 1987 Journal of Applied Physics 6181 ISSN 0021-8979

[28] Teunissen J, Sun A and Ebert U 2014 Journal of Physics D: Applied Physics 47365203 ISSN 0022-3727, 1361-6463

[29] Hagelaar G J M and Pitchford L C 2005 Plasma Sources Science and Technology 14 722-733 ISSN 0963-0252, 1361-6595

[30] Phelps A V and Pitchford L C 1985 Phys. Rev. A 31 29322949

[31] Teunissen J and Ebert U 2016 Plasma Sources Science and Technology 25044005 ISSN 1361-6595

[32] Babaeva N Y, Tereshonok D V and Naidis G V 2016 Plasma Sources Science and Technology 25044008 ISSN 1361-
6595

[33] Pancheshnyi S V and Starikovskii A Y 2004 Plasma Sources Science and Technology 13 B1-B5 ISSN 0963-0252, 1361-6595

[34] Soloviev V R and Krivtsov V M 2009 Journal of Physics D: Applied Physics 42125208 ISSN 0022-3727, 1361-6463

[35] Soloviev V and Krivtsov V 2017 Journal of Physics: Conference Series 927012059 ISSN 1742-6588, 17426596

[36] Naidis G V 1997 Technical Physics Letters 23 493-494 ISSN 1090-6533

[37] Li C, Ebert U and Hundsdorfer W 2010 Journal of Computational Physics 229 200-220 ISSN 0021-9991 